\title{
Effect of Sitagliptin in Type 1 or Type 2 Diabetic Patients with Absolute Insulin Deficiency: A 48 Weeks Observational Study
}

\author{
Eiji Kutoh $^{1,2^{*}}$ and Tadataka Hori ${ }^{1}$ \\ ${ }^{1}$ Biomedical Center, 1-5-8-613, Komatsugawa, Edogawa, Tokyo, Japan. \\ ${ }^{2}$ Department of Internal Medicine, Division of Diabetes and Endocrinology, Gyoda General \\ Hospital, Saitama, Japan. \\ Authors' contributions \\ This work was carried out in collaboration between all authors. Author EK designed the \\ study, performed the statistical analysis, undertaken literature search and wrote the \\ manuscript. Author TH made contributions during the revision of the manuscript. Both \\ authors read and approved the final manuscript.
}

Short Communication

Received $1^{\text {st }}$ April 2013

Accepted 31 ${ }^{\text {st }}$ May 2013

Published $16^{\text {th }}$ June 2013

\section{ABSTRACT}

Aims: The aim of this study is to investigate the long-term efficacy of sitagliptin added to insulin in type 1 or type 2 diabetic patients with absolute insulin deficiency.

Study Design: 48 weeks open-label, observational study.

Place and Duration of Study: Department of Internal Medicine, Gyoda General Hospital, between June 2010 and December 2012.

Methodology: Sitagliptin $25-100 \mathrm{mg} /$ day was added to the ongoing insulin therapy in those without any detectable post-meal C-peptide levels. $\mathrm{HbA1c}$ and other parameters were followed for 48 weeks.

Results: Effective reductions of HbA1c levels were already observed at 12 weeks and sustainable throughout the study period. However, 2 subjects had severe hypoglycemic evens. Post-meal C-peptide remained undetectable with all the subjects. Interestingly, significant increases of body weight were observed.

Conclusion: Sitagliptin as an adjunct to insulin in patients with absolute insulin deficiency may be effective and sustainable for at least 48 weeks, allowing for less intense therapy. However, it should be noted that some patients may have severe 
hypoglycemic events. In spite of the significant glycemic effects of sitagliptin in the setting of this study, endogenous insulin secretory capacity remained absent, suggesting that the glucose lowering effect of this drug may be mediated through GLP-1 independent pathway as well.

Keywords: Incretin based therapy; DPP-4 inhibitors; sitagliptin; insulin-deficient patien; hypoglycemia.

\section{INTRODUCTION}

While sitagliptin has been approved for clinical use in patients with type 2 diabetes (T2DM) in combination with insulin, it has not been studied in insulin-deficient patients, not whether they have strictly-speaking type 1diabetes (T1DM) or T2DM. As its definition, incretin-based therapies (GLP-1 analogues or DPP-4 inhibitors) stimulate insulin secretion [1]. Thus, it is probably not effective with those who have no residual beta-cell function. The possibility of using incretin-based therapies in patients with T1DM is now emerging [2]. As expected, the ideal candidates for this strategy are individuals who still have certain degrees of preserved $\beta$-cell function [3]. In this connection, it was shown that in patients with LADA (Latent Autoimmune Diabetes in Adults), linagliptin, one of the newest DPP-4 inhibitors on the market, was shown to preserve beta-cell function (as measured by the preservation capacity of C-peptide levels) better than glimepiride [4]. Thus, DPP-4 inhibitors may be clinically useful in patients with T1DM who still preserve certain degrees of beta-cell function (e.g. LADA, reference 4).

However, it was reported that sitagliptin as an add-on to insulin was effective and safe in subjects with absolute insulin deficiency in a case series $(n=3,3$ months follow up period reference: 5). The present study investigates the long-term (48 weeks) efficacy, sustainability and safety of sitaglitin added to the ongoing insulin regimen with islet-antibody positive T1DM and T2DM subjects lacking functional beta-cell function. Although these subjects have very different diabetic backgrounds, the common denominator is more that they were absolutely insulin-deficient.

\section{SUBJECTS AND METHODS}

The baseline characteristics of the patients are summarized in Table 1. Inclusion criteria were those with islet-antibody (against glutamic acid decarboxylase and/or insulin) positive T1DM $(n=12)$ or T2DM $(n=8)$ patients who have no functional beta-cell function (post-meal C-peptide levels below detectable range; $<0.1 \mathrm{ng} / \mathrm{ml}$ ) and were in poor glycemic control. The subjects were undertaking intensive insulin treatment (mean insulin dose per day $45.1 \pm 24.9$ units) together with oral hypoglycemic drugs including metformin, pioglitazone or aglucosidase inhibitors (voglibose or miglitol) but glycemic control remained poor ( $\mathrm{HbA} 1 \mathrm{c}$ $8.22 \pm 0.65 \%$ ). The dose of these oral hypoglycemic drugs could be reduced in the case of improved glycemic control. Additionally these subjects were taking some other drugs for hypertension, hyperlipidemia or hyperuricemia. The doses of these drugs were unchanged during this study. 
Table 1. Baseline characteristics of the patients

\begin{tabular}{|c|c|}
\hline age & $52.7 \pm 16.8$ \\
\hline sex (F/M) & $7 / 13$ \\
\hline T1DM/T2DM & $12 / 8$ \\
\hline HbA1c (\%) & $8.22 \pm 0.65$ \\
\hline duration of diabetes (years) & $14.4 \pm 9.3$ \\
\hline insulin dose (per day) & $45.1 \pm 24.9$ \\
\hline BMI & $22.14 \pm 3.82$ \\
\hline & \\
\hline & \\
\hline other oral drugs (n) & 15 \\
\hline metformin & 3 \\
\hline pioglitazone & 8 \\
\hline alpha-glucosidase inhibitors & $1 / 3 / 2$ \\
\hline anti-hypertension (ACE-I/ARB/CCB/ $\alpha$-blocker) & 6 \\
\hline anti-hyperlipidemia (statins) & 1 \\
\hline anti-hyperuricemia (allopurinol) & 3 \\
\hline others & \\
\hline
\end{tabular}

Sitagliptin 25-100 mg/day was added to the regimen. If there was no effect even with the maximum amount (100 mg/day), the subjects were requested to stop sitagliptin. Incidence of hypoglycemia, tolerability, and liver/ kidney functions were monitored and in the case of any clinically significant concerns of these parameters, the subjects were requested to discontinue the project. These drop-out subjects were excluded from the data analysis. The laboratory measurements were performed at the Central Laboratory of Gyoda General Hospital. Informed consents were obtained from the patients and this project has been approved by the ethical committee. The primary efficacy end point was the change of $\mathrm{HbA} 1 \mathrm{c}$ levels from baseline to $12 \mathrm{~W}, 24 \mathrm{~W}, 36 \mathrm{~W}$ and $48 \mathrm{~W}$. HbA1c values were assessed by the JDS standardization [6] throughout this manuscript. To convert these into the National Glycoprotein Standardization Program (NGSP), add approximately $0.4 \%$ to the indicated values. ANOVA (analysis of variance) was used to verify whether statistically significant difference exist between these varying periods of the measured parameters, followed by multiple range test (Dunnett's method). Values of $p<0.05$ were considered significant thought the analysis.

\section{RESULTS}

After addition of sitagliptin, effective reductions of $\mathrm{HbA} 1 \mathrm{c}$ levels were already observed at 12 weeks and this effect was sustainable up to 48 weeks (Fig. 1). While there were no reductions in total daily insulin dose, most subjects could reduce or discontinue some of the oral hypoglycemic drugs. However, 2 subjects had severe hypoglycemic events which occurred within the first month after starting sitagliptin. 2 subjects lost contact 6 month after the start of the study and 1 subject was a non-responder whose HbA1c levels had no changes. These 5 subjects had discontinued the project. 


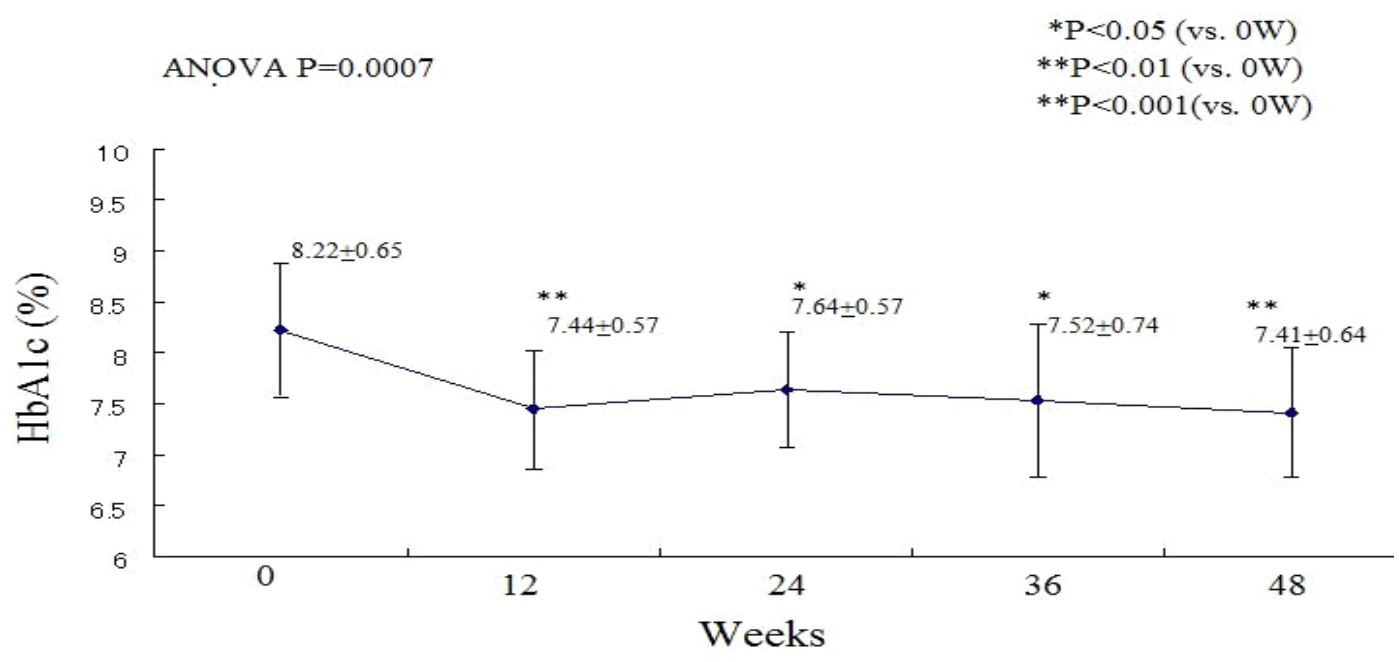

Fig. 1. Change of HbA1c levels with sitagliptin

Sitagliptin was added at week 0 . The change of $H b A 1 \mathrm{c}$ levels was plotted between 0 week (baseline) and up to 48 weeks after the treatment (12W, 24W, 36W and 48W). Data are shown with mean $\pm S D$.

Apart from the severe hypoglycemic events in 2 cases, sitagliptin was well-tolerated and no clinically significant elevations of hepatic or renal parameters were observed. 6 subjects had occasionally mild hypoglycemic events, which could be easily managed by taking candies or glucose drinks by themselves. Surprisingly, C-peptide levels remained repeatedly undetectable after sitagliptin treatment in all the subjects. The C-peptide levels were measured at least twice during the study.

Non-glycemic effects of sitagliptin were also monitored. As shown in Fig. 2, BMI significantly increased at 12 weeks $(22.55 \pm 3.81$ vs. baseline $22.14 \pm 3.82, p<0.02)$ and the levels were unchanged till the end of the study (at 24 weeks: $22.54 \pm 3.29$ v.s. baseline, $p<0.05$; at 48 weeks: $22.58 \pm 3.40$ v.s. baseline, $p<0.01)$.

Other non-glycemic parameters including lipid (T-C, TG, HDL-C and LDL-C) were measured and no significant changes of lipid parameters were observed (results not shown). Blood pressure was also measured, however the variations were so big that no conclusions have been made on this parameter. 


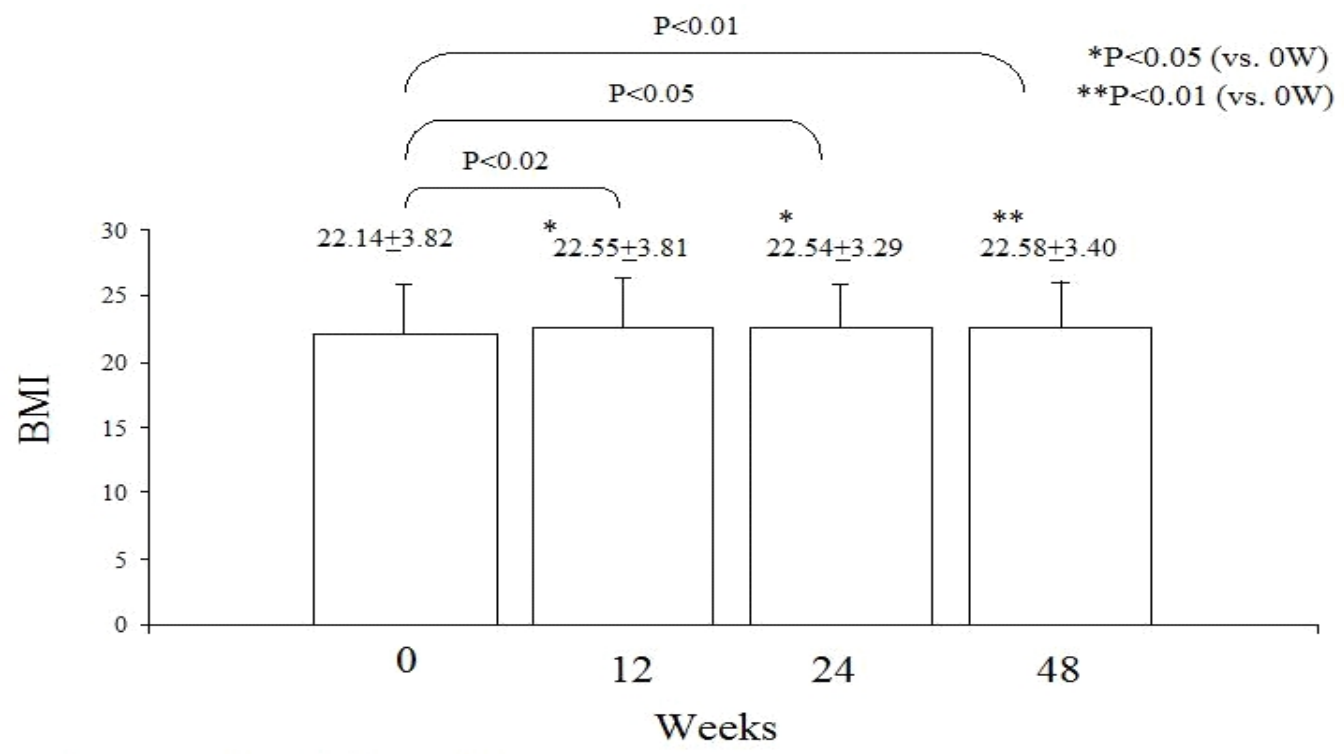

Fig. 2. Change of BMI levels

Sitagliptin was added at week 0 . The change of BMI levels was plotted between 0 week (baseline)

and up to 48 weeks after the treatment (12W, $24 W$, and $48 W$ ). Data are shown with mean $\pm S D$.

\section{DISCUSSION}

In this work, it was shown that sitagliptin added to insulin in patients without residual betacell function is effective and sustainable for at least 48 weeks. However, no improvement of $\beta$-cell function was observed (as measured by the absence of rise in post-meal C-peptide levels). Although the limit of detection of C-peptide in this work was $0.1 \mathrm{ng} / \mathrm{ml}$, one cannot fully exclude the possibility that minimal changes in C-peptide levels may have escaped the analysis. Alternatively, a small peripheral increase, or an intra-islet increase of endogenous insulin levels cannot be ruled out as well.

In these insulin deficient patients, what could have lowered glucose levels without activating beta-cell function? At least three explanations can be postulated. First, many endogenous factor(s) other than GLP-1/GIP might be subject to DPP-4 degradation [7], resulting in their modifications of biological activities. These factors might have effects on glucose homeostasis. DPP-4 inhibitors including sitagliptin may change the ratio of intact/cleaved peptide, thereby resulting in modified glucose levels (Fig. 3A).

Second, the observed glycemic effect in these settings may be due to suppression of glucagon levels. However, it was reported that the glucagon-suppressive effects of sitagliptin (or other DPP-4 inhibitors) are very small and short-lived [5]. Therefore, it is unlikely that reduction of glucagon levels by sitagliptin can fully explain the reduced glucose levels. As a matter of fact, the postprandial glucagon levels before the addition of sitagliptin were within the normal range in some patients who showed significant reductions of glucose levels (results not shown). Third, the glucose-lowering effect of sitagliptin may be mediated through novel mechanisms including GLP-1 independent signal transduction pathways as proposed 
(Fig.3B, reference 5). Further scientific works using molecular and cellular approaches targeting these factors and pathways (indicated in Fig. $3 \mathrm{~A}$ and $3 \mathrm{~B}$ ) will be required to challenge these possibilities.

substrates other than GLP-1

(endocrine peptide, chemokines or neuropeptide etc)

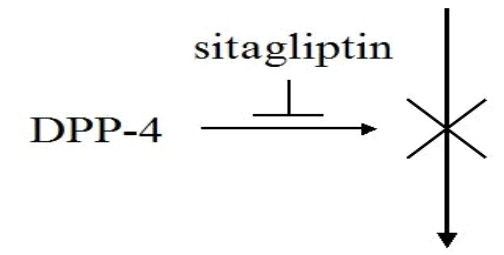

chemically modified above mentioned peptides

resulting in distinct glucose metabolism ??

Fig. 3A. Potential mechanism of sitagliptin in controlling the biological activities of peptides other than GLP-1

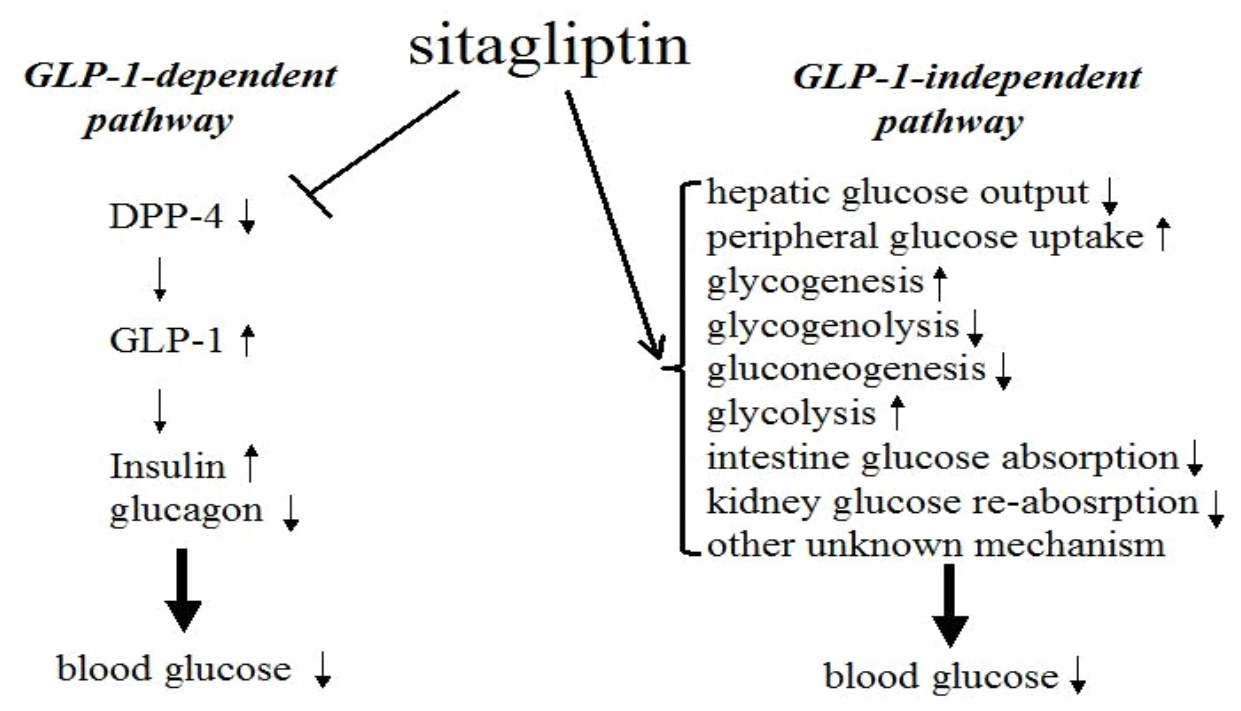

Fig. 3B. GLP-1 dependent and putative GLP-1 independent pathway of sitagliptin

One interesting observation is that significant body weight gain was observed in the setting of this study. This is somehow in contrast to the commonly accepted idea that sitagliptin (or other DPP-4 inhibitors) is weight neutral. There are a number of explanations for this. First, the subjects in this study are absolutely insulin deficient and quite lean (mean BMI is 22.14). Patients who have poor glycemic/metabolic control are usually hypovolemic and underweight. With sitagliptin, the glycemic control improves and consequently, corrections of glycosuria leading to reduced energy loss as well as the increased anabolic effects of insulin (increased lipogenesis) may occur. These backgrounds may have led to body weight gain. Second, sitaliptin is known to increase GIP levels [8], which, in turn, increases body weight $[9,10]$. Involvement of GIP in sitagliptin-medicated body weight gain might be the case. 
Unlike T2DM, leanness or weight loss is a perceived problem in T1DM, thus, weight gain by sitagliptin in insulin deficient, lean patients may be a potential advantage of this drug. Concern has arisen, however, that this weight gain may have adverse effects on the levels of lipid and blood pressure. However, in this study no changes in lipid levels or no consistent results with blood pressured were observed with sitagliptin.

\section{CONCLUSION}

In this study it was implicated that sitagliptin is be effective and sustainable for at least 48 weeks as an adjunct to insulin in those without functional beta-cell function (not whether T1DM or T2DM), allowing for less intense therapy. However it should be noted that some patients may have severe hypoglycemic events, although DPP-4 inhibitors including sitagliptin are known to cause little hypoglycemic events [11]. The underlying mechanism of potential hypoglycemic events induced by sitagliptin remains to be investigated.

In spite of the significant glycemic effects of sitagliptin in the setting of this study, endogenous insulin secretory capacity remained absent, suggesting that the glucose lowering effect of this drug may be mediated through other pathways (involvement of sitagliptin in the regulations of the substrates other than GLP-1 or of GLP-1 independent pathway; as presented in Fig. $3 \mathrm{~A}$ and $3 \mathrm{~B}$ ). In contrast to the commonly accepted idea that DPP-4 inhibitors including sitagliptin is weight neutral, significant body weight gain was observed in the setting of this study. The limitation of this study is that it is an uncontrolled, observational study. Further randomized, double-blind, placebo-controlled longer period studies with increased number of subjects will be necessary to strengthen the findings in this study.

\section{CONSENT}

The authors declare that written informed consent and assent was obtained from the patients.

\section{ETHICAL APPROVAL}

The author hereby declares that all experiments have been examined and approved by the ethics committee by Gyoda General Hospital and have therefore been performed in accordance with the ethical standards laid down in the 1964 Declaration of Helsinki.

\section{ACKNOWLEDGEMENT}

The author thanks Drs. Jan Wajs and Hiroshi Kawashima for supports and discussions.

\section{COMPETING INTERESTS}

The authors declare that no competing interests exist.

\section{REFERENCES}

1. Lovshin JA, Drucker DJ. Incretin-based therapies for type 2 diabetes mellitus. Nat Rev Endocrinol. 2009;5(5):262-269. 
2. Suen CS, Burn P. The potential of incretin-based therapies in type 1 diabetes. Drug Discov Today. 2012;17(1-2):89-95.

3. Bosi E. Time for testing incretin therapies in early type 1 diabetes? J Clin Endocrinol Metab. 2010;95:2607-2609.

4. Johansen OE, Boehm B, Grill V, Torjesen PA, Bhattacharya S, Patel S, Wetzel K, Woerle HJ. $\beta$-cell function in latent autoimmune diabetes in adults treated with linagliptin versus glimepiride: exploratory results from a two year double-blind. Randomized, Controlled Study Diabetes. 2012;61(S1).

5. Kutoh E. Sitagliptin is effective and safe as add-on to insulin in patients with absolute insulin deficiency: a case series. J Med Case Reports. 2011;5:117.

6. Tominaga M, Makino H, Yoshino G, Kuwa K, Takei I, Aono Y, Hoshino T, Umemoto M, Shimatsu A, Sanke T, Kuwashima M, Taminato T, Ono J. Japanese standard reference material_JDS_Lot 2 for haemoglobin A1c. II: Present state of standardization of haemoglobin $A 1 \mathrm{C}$ in Japan using the new reference material in routine clinical assays. Ann Clin Biochem. 2005;42(Pt 1):47-50.

7. Drucker DJ. Dipeptidyl peptidase-4 inhibition and the treatment of type 2 diabetes: preclinical biology and mechanisms of action. Diabetes Care. 2007;30(6):1335-1343.

8. Ahrén B, Hughes TE. Inhibition of dipeptidyl peptidase-4 augments insulin secretion in response to exogenously administered glucagon-like peptide-1, glucose-dependent insulinotropic polypeptide, pituitary adenylate cyclase-activating polypeptide, and gastrin-releasing peptide in mice. Endocrinology. 2005;146(4):2055-2059.

9. $\quad$ Creutzfeldt W. The incretin concept today. Diabetologia. 1979;16(2):75-85.

10. Ugleholdt R. Glucose-dependent Insulinotropic Polypeptide (GIP): From prohormone to actions in endocrine pancreas and adipose tissue. Dan Med Bull. 2011;58(12):B4368.

11. White J. Efficacy and safety of incretin based therapies: clinical trial data. J Am Pharm Assoc. 2009;49(Suppl 1):S30-40.

(C) 2013 Kutoh and Hori; This is an Open Access article distributed under the terms of the Creative Commons Attribution License (http://creativecommons.org/licenses/by/3.0), which permits unrestricted use, distribution, and reproduction in any medium, provided the original work is properly cited.

Peer-review history:

The peer review history for this paper can be accessed here:

http://www.sciencedomain.org/review-history.php?iid=205\&id=12\&aid=1518 